\title{
Dynamic Experiments in Low Vacuum \& ESEM: New Perspectives in Cryo- Electron Microscopy
}

\author{
D.J. Stokes
}

Polymers \& Colloids Group, University of Cambridge, Department of Physics, Cavendish Laboratory, Madingley Road, Cambridge, CB3 0HE, UK

One of the key aspects of low vacuum and environmental scanning electron microscopy is the ability to perform in situ dynamic experiments. Examples include the swelling behavior of textile fibers [1], wetting of polymer substrates [2] and colloidal crystallisation [3], to name but a few. However, very little attention has been paid to the possibilities of extending these methodologies to experiments at low temperatures $\left(-150^{\circ} \mathrm{C}\right.$ to $0^{\circ} \mathrm{C}$, for instance).

Conventional high vacuum cryo-electron techniques for the study of fast-frozen hydrated specimens are well established and extremely useful for studying the 'static' microstructure of organic materials. Meanwhile, the development of in situ 'cryo' experiments, using low vacuum and ESEM technology, will enable observations of dynamic microstructures in a range of interesting systems. This could include structure evolution during freeze-drying, ice crystal nucleation, growth and morphology, controlled etching and sublimation, freeze-thaw or thermal cycling and phase transitions. Applications could include foods, pharmaceutical products, cosmetics, cement and stone, and biological, environmental and atmospheric systems such as frost-forming bacteria, the distribution of ice-adsorbed impurities in clouds and the conditions determining the shapes of snowflakes.

Water vapour is commonly associated with ESEM imaging, particularly because of its useful thermodynamic properties over the range of pressures and temperatures typically used. However, for very low temperature work $\left(<-80^{\circ} \mathrm{C}\right)$, an alternative imaging gas is needed, with a higher vapor pressure than water. Candidates include such gases as $\mathrm{N}_{2} \mathrm{O}$ and $\mathrm{N}_{2}$. Figure 1 demonstrates the use of nitrogen gas to image an uncoated, freeze-fractured specimen at $-95^{\circ} \mathrm{C}$. The very slow rate of sublimation in low vacuum makes it possible to follow changes over an extended time period (several hours in this case), in contrast to the rapid sublimation that would occur in high vacuum [4].

At temperatures warmer than approx $-80^{\circ} \mathrm{C}$, it is possible to maintain small partial pressures of water in the vapor phase. This suggests opportunities for more sophisticated experiments in which two or more gases are present in the chamber, one of which is water vapor: the 'inert' gas continues to serve in its role of providing the image, while water vapor actively participates in the experiment (see Figure 2). This means, for example, that specimens can be stabilised against sublimation at higher temperatures, allowing us to keep frozen specimens intact while getting closer to the temperatures at which changes may occur (particularly relevant to systems with sub-zero glass transition temperatures). By determining a specific set of humidity and thermal criteria, we can also control the freezing/drying of compounds such as those used in drug delivery, giving a direct means of observing the development of porous microstructures. Similar reasoning applies to in situ crystallisation of solutions, where we can study the onset of ice nucleation and follow crystal growth and morphology in the presence or absence of growth-inhibiting additives. These are just a few of the possibilities for new dynamic experiments in electron microscopy. 


\section{References}

[1] L.M. Jenkins and A.M. Donald, Scanning, 19 (1997) 92-97.

[2] N.A. Stelmashenko et al., J. Microscopy, 104(2) (2001) 172-183.

[3] C.B. He and A.M. Donald, Langmuir, 12(26) (1996) 6250-6256.

[4] D.J. Stokes et al., J. Microscopy, (2003 submitted).

[5] This work was funded by a Royal Society Warren Research Fund Dorothy Hodgkin Research Fellowship. The loan of a Gatan Alto 2500 Cryo-transfer system is gratefully acknowledged.
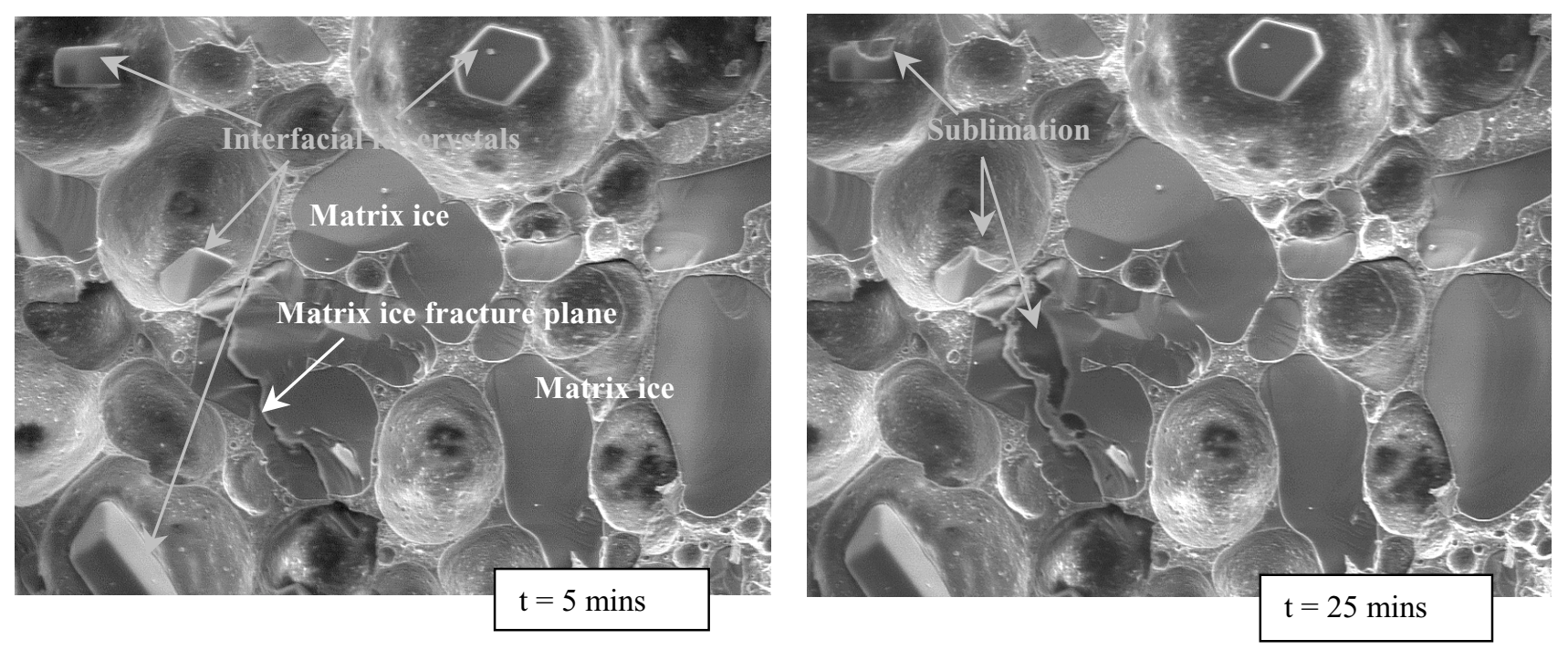

Figure 1. Ice crystals in freeze-fractured ice cream, imaged in $\mathrm{N}_{2}$ at $\mathrm{T}=-95^{\circ} \mathrm{C}$. The low vacuum environment confers considerable stability to the specimen, enabling controlled in situ etching over an extended time period.

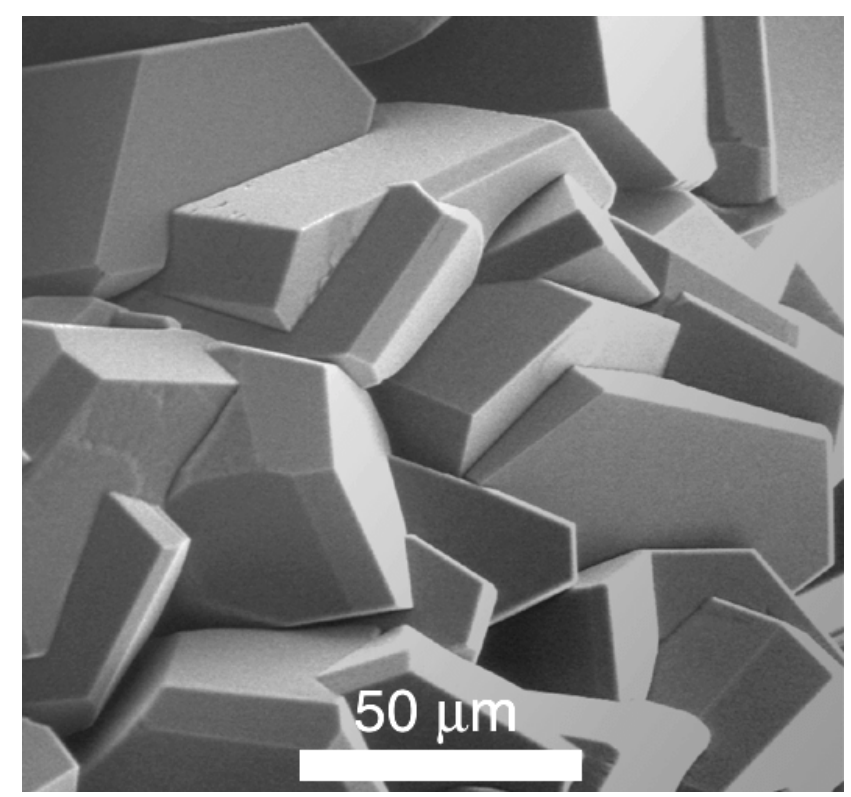

Figure 2. Mixing gases in ESEM: $\mathrm{N}_{2} / \mathrm{H}_{2} \mathrm{O}$ at $\mathrm{T}=-65^{\circ} \mathrm{C}$. Conditions are just sufficient for vapor deposition of $\mathrm{H}_{2} \mathrm{O}$ ice crystals, while $\mathrm{N}_{2}$ remains in the vapor phase, forming the image. 